\title{
A General Stability Result for Viscoelastic Equations with Singular Kernels \\ Messaoudi $\mathrm{SA}^{1 *}$ and Khenous $\mathrm{HB}^{2}$
}

${ }^{1}$ King Fahd University of Petroleum and Minerals, Department of Mathematics and Statistics, Saudi Arabia

${ }^{2}$ King Khalid University, Department of Mathematics, Saudi Arabia

\begin{abstract}
Viscoelasticity with regular relaxation functions has attracted the attention of many researchers over the last half century or so. Several results concerning existence and long-time behavior of solutions have been established. In particular the exponential, polynomial decay and recently what so called the general decay have been proved.

For viscoelasticity, with singular kernels, less attention has been given and few results of existence and exponential decay have been established. In this paper we extend the general decay result, established for regularkernel viscoelasticity, to that with singular kernels. We also present some numerical test to illustrate our theoretical result.
\end{abstract}

Keywords: General decay; Viscoelastic damping; Singular relaxation function

AMS Classification: 35B37, 35L55, 74D05, 93D15.

\section{Introduction}

Since the pioneer work of Dafermos [1,2] in 1970, the viscoelastic equation

$$
u_{t t}-\Delta u+\int_{0}^{t} g(t-s) \Delta u(s) d s=0,
$$

with smooth kernel, has attracted a great deal of researchers and several existence and stability results have been established. See, for instance, the works of [3-19] where the relaxation function was assumed to be either of polynomial or of exponential decay. Messaoudi [20] studied (1.1) for more general decaying kernels and established some general decay results, from which the usual exponential and polynomial rates are only special cases. After that a series of papers have appeared, using similar techniques, and obtaining similar general decay results. See, among others, [21-25].

Following the work by Mustafa and Messaoudi [26], Lasiecka et al. discussed (1.1) with a relaxation function satisfying

$$
g^{\prime}(s)+H(g(s)) \leq 0, \forall s \geq 0,
$$

Where $\mathrm{H}$ is a given continuous positive increasing convex function such that $\mathrm{H}(0)=0$, and developed an intrinsic method for determining optimal decay rates.

In all the above mentioned works, kernels are assumed to be regular on $\%[0,+\infty)$.

However, Kinetic theories for chain molecules as mentioned in [27] and some experimental data [28] suggests that this a realistic possibility, at least for some viscoelastic materials like dilute solutions of coiling polymers. Contrary to the regular kernel case, only very few results related to singular (at the origin) kernels have been established. For instance, Hrusa and Renardy [29] studied a model equation in nonlinear viscoelasticity and proved local and global existence theorems, allowing the memory function to have a singularity. To achieve their result, they approximated the equation by equations with regular kernels and then used the energy estimates to prove convergence of the approximate solutions. In [30], the authors showed that a singular kernel may yield smoothing effects for the solution of an evolution prob- lem, though the gain in regularity cannot be derived without specifying the kind of singularity [31]. Gentili considered a linear viscoelastic material with a relaxation function which may exhibit an initial singularity. He used the Laplace transform to study existence, uniqueness and asymptotic behavior of the solution to the dynamic problem. To provide these results, the author required the relaxation function to satisfy only restrictions deriving from Thermodynamics. He also used the energy method to establish a stability theorem and obtained a regularity result for a class of singular kernels which ensures the asymptotic stability of the solution. Tatar considered

$$
u_{t t}-\Delta u-\Delta u_{t t}+\int_{0}^{t} g(t-s) \Delta u(s) d s=0,
$$

together with initial and Dirichlet-boundary conditions and for a relaxation function

$$
g(t)=t^{-\alpha} e^{-\beta t} / \Gamma(1-\alpha), 0<\alpha<1, \% \beta>0
$$

and proved an exponential decay result. Notice that the kernel here exhibits an initial singularity, summable, and decays exponentially at infinity. This type of kernels appears mostly in fractional calculus [32]. $\mathrm{Wu}$ [33] extended Tatar's result to the equation

$$
\left|u_{t}\right|^{\rho} u_{t t}-\Delta u-\Delta u_{t t}+\int_{0}^{t} g(t-s) \Delta u(s) d s+|u|^{p} u=0,
$$

with $\rho, p>0$ and $\mathrm{g}$ in [34]. We refer the reader to Carillo et al. [35-37] for more recent results regarding viscoelastic problems with singular Kernels.

In this paper we are concerned with the following viscoelastic problem

$$
\left\{\begin{array}{lr}
u_{t t}-\Delta u+\int_{0}^{t} g(t-s) \Delta u(s) d s=0, & \text { in } \Omega \times(0, \infty) u=0, \\
u=0, & \text { on } \partial \Omega \times(0, \infty) u(x, 0)=u_{0}(x), \\
u(x, 0)=u_{0}(x), u_{t}(x, 0)=u_{1}(x), & x \in \Omega,
\end{array}\right.
$$

${ }^{*}$ Corresponding author: Messaoudi SA, King Fahd University of Petroleum and Minerals, Department of Mathematics and Statistics, Saudi Arabia, Tel: +966-72291379; E-mail: messaoud@kfupm.edu.sa

Received March 24, 2015; Accepted May 13, 2015; Published May 20, 2015

Citation: Messaoudi SA, Khenous HB (2015) A General Stability Result for Viscoelastic Equations with Singular Kernels. J Appl Computat Math 4: 221. doi:10.4172/2168-9679.1000221

Copyright: ( 2015 Messaoudi SA, et al. This is an open-access article distributed under the terms of the Creative Commons Attribution License, which permits unrestricted use, distribution, and reproduction in any medium, provided the original author and source are credited. 
Where $\Omega$ is a bounded domain of $\mathrm{R}^{\mathrm{n}}(\mathrm{n} \geq 1)$ with a smooth boundary $\partial \Omega$ and the relaxation function $g$ is a positive non increasing function which can exhibit a singularity at 0 . Our aim in this work is to show that, with a slight modification in the arguments of [20], we extend the general decay result, established for regular relation functions, to singular kernels. As we show later, the exponential decay results of Tatar [31] and $\mathrm{Wu}$ [27], among others, are only special cases. We also give some examples and present some numeric to illustrate our decay results. The paper is organized as follows. In section 2, we present some hypotheses and technical lemmas and our main decay result. In section 3 , the proof of our main result, as well as some illustrating examples will be given. Section 4 is devoted to the numerical setting and tests of our decay results. We finish our paper with some concluding remarks [11].

\section{Preliminaries}

In this section we state our hypotheses, give, without proof a standard existence theorem, and state our main decay result. So, we assume

(H1) g: $(0,+\infty) \rightarrow(0,+\infty)$ is a differentiable integrable function satisfying

$$
1-\int_{0}^{+\infty} g(s) d s=l>0
$$

$(\mathrm{H} 2)$ There exists a differentiable non increasing function $\xi:(0,+\infty)$ $\rightarrow(0,+\infty)$ such that

$$
g^{\prime}(t) \leq-\xi(t) g(t), \quad \forall t>0 .
$$

Remark 2.1: These conditions allow a larger class of functions than that considered in $[14,20]$ and others. In particular it allows singular integrable functions such as

$$
g(t)=t^{-a} e^{-b t^{\nu}}, 0<a<1,0<v \leq 1, b>0 .
$$

Notice, also, that $\left|\xi^{\prime}(t) / \xi(t)\right| \leq M$ imposed in $[14,20]$ is no longer required. Now, we introduce the energy functional

$$
E(t):=\frac{1}{2} \int_{\Omega}\left(u_{t}^{2}+\left(1-\int_{0}^{t} g(s) d s\right)|\nabla u|^{2}\right) d x+\frac{1}{2}\left(g^{\circ} \nabla u\right)(t),
$$

Where

$$
\left(g^{\circ} \nabla u\right)(t)=\int_{\Omega} \int_{0}^{t} g(t-s)|\nabla u(t)-\nabla u(s)|^{2} d s d x .
$$

For completeness, we state the following existence result, which can be proved using the Galerkin method [7]. For more about existence, see [38] and [29].

Proposition 2.1: Let $\left(u_{0}, u_{1}\right) \in H_{0}^{1}(\Omega) \times L^{2}(\Omega)$ be given. Assume that $g$ satisfies (G1). Then problem (1.2) has a unique global solution

$$
u \in C\left([0,+\infty) ; H_{0}^{1}(\Omega)\right), \quad u_{t} \in C\left([0,+\infty) ; L^{2}(\Omega)\right) .
$$

Our main stability result is

Theorem 2.2: Assume that (H1) and (H2) hold. Then, for any $\mathrm{t}_{0}>0$, there exist two positive constants $\mathrm{k}$ and $\mathrm{K}$ such that the solution of (1.2) satisfies

$$
E(t) \leq K e^{-k \int_{10}^{t} \xi(s) d s} \quad \forall t \geq t_{0} .
$$

\section{Main Result}

In this section we prove our main decay result. We will use c, throughout this paper, to denote a generic positive constant. We start with the following lemmas.

Lemma 2.2: [20] Let $u$ be the solution of (1.2). Then the energy functional satisfies

$$
E^{\prime}(t)=\frac{1}{2}\left(g^{\circ} \nabla u\right)-\frac{1}{2} \% g(t) \int_{\Omega}|\nabla u|^{2} d x \leq 0 .
$$

Lemma 2.3: [20] For any $u \in H_{0}^{1}(\Omega)$, we have

$$
\int_{\Omega}\left(\int_{0}^{t} g(t-\tau)(u(t)-u(\tau)) d \tau\right)^{2} d x \leq(1-l) C_{p}^{2}\left(g^{\circ} \nabla u\right)(t),
$$

$\mathrm{C}_{\mathrm{p}}$ is the Poincar'e constant.

Lemma 2.4: Under the assumption (H1) and (H2), the functional $\Phi(t):=\int_{\Omega} u u_{t} d x$

Satisfies, along the solution, the estimate

$\Phi^{\prime}(t) \leq-\frac{l}{2} \int_{\Omega}|\nabla u|^{2}+\int_{\Omega} u_{t}^{2}+c\left(g^{\circ} \nabla u\right)$.

Proof: By using equation (1.2), we easily see that

$$
\Phi^{\prime}(t)=\int_{\Omega} u_{t}^{2} d x-\left.\int_{\Omega} \nabla u\right|^{2} d x+\int_{\Omega} \nabla u(t) \cdot \int_{0}^{t} g(t-\tau) \nabla u(\tau) d \tau d x .
$$

We then estimate the third term in the right-hand side of (3.3), as in Lemma 3.4 [20], to obtain

$$
\begin{aligned}
& \int_{\Omega} \nabla u(t) \cdot \int_{0}^{t} g(t-\tau) \nabla u(\tau) d \tau d x \leq\left.\frac{1}{2} \int_{\Omega} \nabla u(t)\right|^{2} d x \\
& +\frac{1}{2}\left[\left(1+\frac{1}{\eta}\right)(1-l)\left(g^{\circ} \nabla u\right)(t)+\left.(1+\eta)(1-l)^{2} \int_{\Omega} \nabla u(t)\right|^{2} d x\right], \forall \eta>0 .
\end{aligned}
$$

By combining (3.3) and (3.4), we arrive at

$$
\Phi^{\prime}(t) \leq \int_{\Omega} u_{t}^{2} d x+\frac{1}{2}\left(1+\frac{1}{\% \eta}\right)(1-l)\left(g^{\circ} \nabla u\right)(t)-\left.\frac{1}{2}\left[1-(1+\eta)(1-l)^{2}\right] \int_{\Omega} \nabla u(t)\right|^{2} d x, \forall \eta>0
$$

By choosing $\eta=l /(1-l),(3.2)$ is established.

Lemma 2.5: Under the assumption (H1) and (H2), the functional

$$
\Psi(t):=-\int_{\Omega} u_{t} \int_{t_{0}}^{t} g(t-s)(u(t)-u(s)) d s d x
$$

With $\mathrm{t}_{0}>0$ satisfies, along the solution and for any $\delta>0$, the estimate

$$
\Psi^{\prime}(t) \leq-\left(\int_{t_{0}}^{t} g(s) d s-\delta\right) \int_{\Omega} u_{t}^{2}+\delta \int_{\Omega}|\nabla u|^{2}+\% \frac{c}{\delta}\left(g^{\circ} \nabla u\right)-\frac{c}{\delta}\left(g^{\prime} \nabla u\right) .
$$

Proof: Direct computations, using (1.2), yield

$$
\begin{aligned}
& \Psi^{\prime}(t)=\int_{\Omega} \nabla u(t) \cdot\left(\int_{t_{0}}^{t} g(t-\tau)(\nabla u(t)-\nabla u(\tau)) d \tau\right) d x \\
& -\int_{\Omega}\left(\int_{0}^{t} g(t-\tau) \nabla u(\tau) d \tau\right) \cdot\left(\int_{t_{0}}^{t} g(t-\tau)(\nabla u(t)-\nabla u(\tau)) d \tau\right) d x \\
& -\int_{\Omega} u_{t} \int_{t_{0}}^{t} g^{\prime}(t-\tau)(u(t)-u(\tau)) d \tau d x-\left(\int_{t_{0}}^{t} g(s) d s\right) \int_{\Omega} u_{t}^{2} d x
\end{aligned}
$$

Similarly to (3.3), we estimate the right-hand side terms of (3.7). So, by Lemma 2.3 , Young's inequality and the fact that

$$
\int_{t_{0}}^{t} g(\tau) d \tau \leq \int_{0}^{+\infty} g(\tau) d \tau=1-l
$$

the first term of (3.7) gives

$$
\begin{aligned}
& -\int_{\Omega} \nabla u(t) \cdot\left(\int_{t_{0}}^{t} g(t-\tau)(\nabla u(t)-\nabla u(\tau)) d \tau\right) d x \\
& \leq\left.\delta \int_{\Omega} \nabla u\right|^{2} d x+\frac{1-l}{4 \delta}\left(g^{\circ} \nabla u\right)(t), \quad \forall \delta>0 .
\end{aligned}
$$

Similarly to (3.4), the second term of (3.7) can be estimated as follows 


$$
\begin{aligned}
& \int_{\Omega}\left(\int_{0}^{t} g(t-s) \nabla u(s) d s\right) \cdot\left(\int_{t_{0}}^{t} g(t-s)(\nabla u(t)-\nabla u(s)) d s\right) d x \\
& \leq\left(2 \delta+\frac{1}{4 \delta}\right)(1-l)\left(g^{\circ} \nabla u\right)(t)+2 \delta(1-l)^{2} \int_{\Omega}|\nabla u|^{2} d x .
\end{aligned}
$$

As for the third and the fourth terms of (3.7) we have

$$
\begin{aligned}
& -\int_{\Omega} u_{t} \int_{t_{0}}^{t} g^{\prime}(t-\tau)(u(t)-u(\tau)) d \tau d x \\
& \leq \delta \int_{\Omega}\left|u_{t}\right|^{2} d x-\frac{g\left(t_{0}\right)}{\% 4 \delta} C_{p}^{2} \int_{t_{0}}^{t} g^{\prime}(t-\tau)|\nabla u(t)-\nabla u(\tau)|^{2} d \tau d x \\
& \leq \delta \int_{\Omega}\left|u_{t}\right|^{2} d x-\frac{g\left(t_{0}\right)}{\% 4 \delta} C_{p}^{2}\left(g^{\prime} \nabla u\right)(t)
\end{aligned}
$$

By combining (3.7)-(3.10), the assertion of the lemma is established.

Proof of Theorem 2.2: We set

$\mathrm{F}(\mathrm{t}):=\mathrm{NE}(\mathrm{t})+\varepsilon \Phi(\mathrm{t})+\Psi(\mathrm{t})$,

where $\mathrm{N}$ and $\varepsilon$ are positive constants. Direction substitution leads to

$$
F^{\prime}(t) \leq-\left[\left(\int_{t_{0}}^{t} g(s) d s-\delta\right)-\varepsilon\right] \int_{\Omega} u_{t}^{2} d x-\left[\frac{l \varepsilon}{2}-\delta\right] \int_{\Omega}|\nabla u|^{2}+\left[\frac{N}{2}-\frac{c}{\delta}\right]\left(g^{\circ} \nabla u\right)+c\left(g^{\circ} \nabla u\right) \cdot c
$$

Since $\mathrm{g}(\mathrm{s})>0$, we can choose $\mathrm{t}_{1}>\mathrm{t}_{0}$ such that $\int_{t_{0}}^{t_{1}} g(s) d s=g_{0}>0$. Hence, we obtain

$$
F^{\prime}(t) \leq-\left[\left(g_{0}-\delta\right)-\varepsilon\right] \int_{\Omega} u_{t}^{2} d x-\left[\frac{l \varepsilon}{2}-\delta\right] \int_{\Omega}|\nabla u|^{2}+\left[\frac{N}{2}-\frac{c}{\delta}\right]\left(g^{\prime} \nabla u\right)+c\left(g^{\circ} \nabla u\right), \forall t \geq t_{1} .
$$

At this point, we choose $\delta$ so small that

$g_{0}-\delta>\frac{1}{2} g_{0}, \delta<\frac{l}{8} g_{0}$

And

$$
\frac{g_{0}}{4}<\varepsilon<\frac{g_{0}}{2}
$$

So that

$$
\left(g_{0}-\delta\right)-\varepsilon>0, \frac{l \varepsilon}{2}-\delta>0 .
$$

Finally, we then pick N so large that $\frac{N}{2}-\frac{c}{\delta} \geq 0$ and $F(t) \backsim E(t)$. Therefore, we have, for some $\gamma>0$,

$$
F^{\prime}(t) \leq-\gamma E(t)+c\left(g^{\circ} \nabla u\right), \forall t \geq t_{1} .
$$

By using (H2) and (3.12), we easily deduce

$$
\begin{aligned}
& (\xi(t) F(t))^{\prime}=\xi(t) F^{\prime}(t)+\xi^{\prime}(t) F(t) \\
& \leq-\gamma \xi(t) E(t)+c\left(g^{\prime} \nabla u\right), \forall t \geq t_{1},
\end{aligned}
$$

Since $\xi^{\prime} \leq 0$. By recalling $F(t) \odot E(t)$, we get

$L^{\prime}(t) \leq-\gamma \xi(t) E(t) \leq-k \xi(t) L(t), \forall t \geq t_{1}$

Where

$$
\begin{aligned}
& L(t)=\xi(t) F(t)+c E(t) \odot E(t) . \\
& L(t) \leq K e^{-k \int_{t_{1}}^{t} \xi(s) d s} \quad \forall t \geq t_{1} .
\end{aligned}
$$

Then, (2.4) follows by (3.14) and continuity of $\xi$ and E.

Decay examples. To illustrate our result, we give four examples of singular relaxation functions.

\section{Let}

$$
g(t)=c_{0} t^{-a} e^{-b t}, 0<a<1, b>0,
$$

And $\mathrm{c}_{0}$ small enough o that $\int_{0}^{+\infty} g(t)<1$ Direct calculations show that $\xi(\mathrm{t})=\mathrm{b}+\mathrm{at}^{-1}$. So, the decay estimate (2.4) gives

$\mathrm{E}(\mathrm{t}) \leq \mathrm{K} \mathrm{t}^{-\mathrm{ak}} \mathrm{e}^{-\mathrm{bkt}}$,

for two positives constants $\mathrm{k}$ and $\mathrm{K}$.

2. Let

$$
\mathrm{g}(\mathrm{t})=\mathrm{c}_{0}[\ln (1+\mathrm{t})]^{-\mathrm{ae}-\mathrm{bt}}, 0<\mathrm{a}<1, \mathrm{~b}>0,
$$

and $\mathrm{c}_{0}$ small enough so that $\int_{0}^{+\infty} g(t)<1$. Direct calculations show that $\xi(t)=b+\frac{a}{(1+t) \ln (1+t)}$. So, the decay estimate (2.4) gives

$$
\mathrm{E}(\mathrm{t}) \leq \mathrm{K}[\ln (1+\mathrm{t})]^{-\mathrm{ak}} \mathrm{e}^{-\mathrm{bkt}},
$$

for two positives constants $\mathrm{k}$ and $\mathrm{K}$.

3. Let $g(t)=c_{0} \frac{e^{-\sqrt{t}}}{\sqrt{t}}$, forc $c_{0}$, for $\mathrm{c} 0$ small enough so that $\int_{0}^{+\infty} g(t)<1$. Direction calculation show that $\xi(t)=\frac{1}{2 t}+\frac{1}{2 \sqrt{t}}$. So, the decay estimate (2.4) gives

$E(t) \leq K t^{-k / 2} e^{-k \sqrt{t}}$,

for two positives constants $\mathrm{k}$ and $\mathrm{K}$.

4. Let

$\mathrm{g}(\mathrm{t})=\mathrm{c}_{0}(1+\mathrm{t})^{-\mathrm{b}}(\ln (1+\mathrm{t}))^{-\mathrm{a}}, 0<\mathrm{a}<1, \mathrm{~b}>1$,

and $\mathrm{c}_{0}$ small enough so that $\int_{0}^{+\infty} g(t)<1$. Direction calculations show that $\xi(t)=\frac{b}{1+t}+\frac{a}{(1+t) \ln (1+t)}$. So, the decay estimate (2.4) gives

$\mathrm{E}(\mathrm{t}) \leq \mathrm{K}(1+\mathrm{t})^{-\mathrm{kb}}(\ln (1+\mathrm{t}))^{-\mathrm{ka}}$,

for two positives constants $\mathrm{k}$ and $\mathrm{K}$.

\section{Numerical Test}

In this section, we present a two dimensional case of system (1.1) in order to illustrate our theoretical decay result.

\section{Numerical scheme}

For computational purposes, we rewrite (1.1) as follows

$u_{t t}-\left(1-\int_{0}^{t} g(s) d s\right) \Delta u-\int_{0}^{t} g(t-s)(\Delta u(s)-\Delta u(t)) d s=0$.

We consider a square domain $[0,1] \times[0,1]$ meshed uniformly in $\mathrm{N}_{\mathrm{x}} \times \mathrm{N}_{\mathrm{y}}$ grids with the space steps $\Delta \mathrm{x}=\Delta \mathrm{y}=1 / \max \left(\mathrm{N}_{\mathrm{x}}, \mathrm{N}_{\mathrm{y}}\right)$. We chose a time interval $[0, T]$ subdivided uniformly into $\mathrm{N}_{\mathrm{t}}=\mathrm{T} / \Delta \mathrm{t}$ sub-intervals with a time step $\Delta \mathrm{t}=\alpha \Delta \mathrm{x}$. The solution $\mathrm{u}(\mathrm{x}, \mathrm{y}, \mathrm{t})$ approximated at each point of the mesh $\left(\mathrm{x}_{\mathrm{i}}, \mathrm{y}_{\mathrm{j}}\right)=(\mathrm{i} \Delta \mathrm{x}, \mathrm{j} \Delta \mathrm{y})$ and at any time $\mathrm{t}^{\mathrm{n}}=\mathrm{n} \Delta \mathrm{t}$ is given by $U_{i, j}^{n} \approx u\left(x_{i}, y_{j}, t^{n}\right)=u_{i, j}^{n}$. At each time $\mathrm{t}^{\mathrm{n}}$ the interval $\left[0, \mathrm{t}^{\mathrm{n}}\right]$ is subdivided uniformly in $\mathrm{n}$ sub-intervals using the same time step $\Delta \mathrm{t}$ where the function $\mathrm{g}$ at each time $\mathrm{s}^{\mathrm{k}}=\mathrm{k} \Delta \mathrm{t}$ is given by $\mathrm{g}\left(\mathrm{s}^{\mathrm{k}}\right)$.

The full discretization of (1.1) in time and space is given by

$\Delta_{t} U_{i, j}^{n}-l \Delta U_{i, j}^{n}+\int_{0}^{t^{n}} g\left(t^{n}-s\right)\left(\Delta U_{i, j}(s)-\Delta U_{i, j}^{n}\right) d s=0$,

With the boundary condition

$U_{0, j}^{n}=U_{N_{x}, j}^{n}=U_{i, 0}^{n}=U_{i, N_{y}}^{n}=0, \forall 0 \leq i, j \leq N_{x}, N_{y}$

And the initial conditions 
$U_{i, j}^{0}=\sin \left(\pi x_{i}\right) \sin \left(\pi y_{j}\right), \frac{\% U_{i, j}^{1}-U_{i, j}^{0}}{\Delta t}=1, \forall 0 \leq i, j \leq N_{x}, N_{y}$,

Where

$$
\begin{aligned}
& \Delta_{t} U_{i, j}^{n}=\frac{U_{i, j}^{n+1}-2 U_{i, j}^{n}+U_{i, j}^{n-1}}{\%(\Delta t)^{2}}, l=1-\int_{0}^{t^{n}} g(s) d s \\
& \Delta U_{i, j}^{n}=\frac{U_{i+1, j}^{n}-2 U_{i, j}^{n}+U_{i-1, j}^{n}}{(\Delta x)^{2}}-\frac{U_{i, j+1}^{n}-2 U_{i, j}^{n}+U_{i, j-1}^{n}}{(\Delta y)^{2}}
\end{aligned}
$$

And

$$
\Delta U_{i, j}(s)=\frac{U_{i+1, j}(s)-2 U_{i, j}(s)+U_{i-1, j}(s)}{(\Delta x)^{2} \%}+\frac{U_{i, j+1}(s)-2 U_{i, j}(s)+U_{i, j-1}(s)}{(\Delta y)^{2}} .
$$

Then, the proposed scheme to solve problem (1.1) is as follows:

$$
\begin{aligned}
& \forall i=0, \ldots, N_{x^{\prime}} \forall j=0, \ldots, N_{y}, \forall n=1, \ldots, N_{t}-1: \\
& U_{i, j}^{n+1}=2 U_{i, j}^{n}-U_{i, j}^{n-1}+(\Delta t)^{2}\left(l \Delta U_{i, j}^{n}+\int_{0}^{t^{n}} g\left(t^{n}-s\right)\left(\Delta U_{i, j}^{n}-\Delta U_{i, j}(s)\right) d s\right) .
\end{aligned}
$$

\section{Consistency-Global truncation error}

The global truncation error $e_{i, j}^{n}$ is obtained by replacing the approximate solution $U_{i, j}^{n}$ by the value of the exact solution $\mathrm{u}(\mathrm{x}, \mathrm{y}, \mathrm{t})$ at the point $\left(x_{i}, y_{j}, t^{n}\right)\left(u\left(x_{i}, y_{j}, t^{n}\right)=u_{i, j}^{n}\right)$ in the scheme (4.2) as follows:

$$
e_{i, j}^{n}=\Delta_{t} u_{i, j}^{n}-\left(1-\int_{0}^{t^{n}} g(s) d s\right) \Delta u_{i, j}^{n}+\int_{0}^{t^{n}} g\left(t^{n}-s\right)\left(\Delta u_{i, j}(s)-\Delta u_{i, j}^{n}\right) d s \neq 0 .
$$

Suppose that $\mathrm{u}$ is at least $\mathrm{C}^{2}$ and compute the Taylor approximation of the second derivative of $\mathrm{u}$ with respect to $\mathrm{t}, \mathrm{x}$ and $\mathrm{y}$ respectively:

$$
\begin{aligned}
& \Delta_{t} u_{i, j}^{n}=\frac{u_{i, j}^{n+1}-2 u_{i, j}^{n}+u_{i, j}^{n-1}}{\Delta t^{2}}=\frac{\partial^{2} u_{i, j}^{n}}{\partial t^{2}}+\frac{2}{4 !} \Delta t^{2} \frac{\partial^{4} u_{i, j}^{n}}{\partial t^{4}}+O\left(\Delta t^{4}\right), \\
& \frac{u_{i+1, j}^{n}-2 u_{i, j}^{n}+u_{i-1, j}^{n}}{\Delta x^{2}}=\frac{\partial^{2} u_{i, j}^{n}}{\partial x^{2}}+\frac{2}{4 !} \Delta x^{2} \frac{\partial^{4} u_{i, j}^{n}}{\partial x^{4}}+O\left(\Delta x^{4}\right), \\
& \frac{u_{i, j+1}^{n}-2 u_{i, j}^{n}+u_{i, j-1}^{n}}{\Delta y^{2}}=\frac{\partial^{2} u_{i, j}^{n}}{\partial y^{2}}+\frac{2}{4 !} \Delta y^{2} \frac{\partial^{4} u_{i, j}^{n}}{\partial y^{4}}+O\left(\Delta y^{4}\right), \\
& \Delta u_{i, j}^{n}=\frac{\partial^{2} u_{i, j}^{n}}{\partial x^{2}}+\frac{\partial^{2} u_{i, j}^{n}}{\partial y^{2}}+\frac{2}{4 !}\left(\Delta x^{2} \frac{\partial^{4} u_{i, j}^{n}}{\partial x^{4}}+\Delta y^{2} \frac{\partial^{4} u_{i, j}^{n}}{\partial y^{4}}\right)+O\left(\Delta x^{4}+\Delta y^{4}\right), \\
& \Delta_{i, j}(s)=\frac{\partial^{2} u_{i, j}(s)}{\partial x^{2}}+\frac{\partial^{2} u_{i, j}(s)}{\partial y^{2}}+\frac{2}{4 !}\left(\Delta x^{2} \frac{\partial^{4} u_{i, j}(s)}{\partial x^{4}}+\Delta y^{2} \frac{\partial^{4} u_{i, j}(s)}{\partial y^{4}}\right)+O\left(\Delta x^{4}+\Delta y^{4}\right) .
\end{aligned}
$$

By substituting (4.7)-(4.8)-(4.9) into (4.6), we find

$$
\begin{aligned}
& e_{i, j}^{n}=\frac{\partial^{2} u_{i, j}^{n}}{\partial t^{2}}-\left(1-\int_{0}^{t^{n}} g(s) d s\right) \Delta u_{i, j}^{n}+\int_{0}^{t^{n}} g\left(t^{n}-s\right)\left(\Delta u_{i, j}(s)-\Delta u_{i, j}^{n}\right) d s \\
& +\frac{1}{12}\left[\Delta t^{2} \frac{\partial^{4} u_{i, j}^{n}}{\partial t^{4}}+\Delta x^{2}\left(\frac{\partial^{4} u_{i, j}^{n}}{\partial x^{4}}+\int_{0}^{t^{n}} g\left(t^{n}-s\right) \frac{\partial^{4} u_{i, j}(s)}{\partial x^{4}} d s\right)\right. \\
& \left.+\Delta y^{2}\left(\frac{\partial^{4} u_{i, j}^{n}}{\partial y^{4}}+\int_{0}^{t^{n}} g\left(t^{n}-s\right) \frac{\partial^{4} u_{i, j}(s)}{\partial y^{4}} d s\right)\right]+O\left(\Delta t^{4}+\Delta x^{4}+\Delta y^{4}\right) .
\end{aligned}
$$

But the first term in the right hand side vanishes, using problem (1.1). Hence, we obtain

$$
\begin{aligned}
& e_{i, j}^{n}=\frac{1}{12}\left[\Delta t^{2} \frac{\partial^{4} u_{i, j}^{n}}{\partial t^{4}}+\Delta x^{2}\left(\frac{\partial^{4} u_{i, j}^{n}}{\partial x^{4}}+\int_{0}^{t^{n}} g\left(t^{n}-s\right) \frac{\partial^{4} u_{i, j}(s)}{\partial x^{4}} d s\right)\right. \\
& \left.+\Delta y^{2}\left(\frac{\partial^{4} u_{i, j}^{n}}{\partial y^{4}}+\int_{0}^{t^{n}} g\left(t^{n}-s\right) \frac{\partial^{4} u_{i, j}(s)}{\partial y^{4}} d s\right)\right]+O\left(\Delta t^{4}+\Delta x^{4}+\Delta y^{4}\right) . \\
& O\left(\Delta t^{2}+\Delta x^{2}+\Delta y^{2}\right)
\end{aligned}
$$

We conclude that scheme (4.5) is consistent of order 2 in time and in space.

\section{Stability-Estimation of the discrete energy}

The discrete energy of system (4.2)-(4.4) is given by

$$
\begin{aligned}
& E^{n+1 / 2}=\frac{1}{2} \sum_{i, j}\left|\frac{U_{i, j}^{n+1}-U_{i, j}^{n}}{\% \Delta t}\right|^{2} \Delta x \Delta y+\frac{1}{2}\left(1-\int_{0}^{t^{n}} g(s) d s\right) \sum_{i, j} \Delta U_{i, j}^{n} U_{i, j}^{n+1} \Delta x \Delta y \\
& +\frac{1}{2} \sum_{k=0}^{n} g\left(t^{n}-s^{k}\right) \sum_{i, j}\left(-\Delta U_{i, j}^{n}+\Delta U_{i, j}^{k}\right) U_{i, j}^{n+1} \Delta t \Delta x \Delta y .
\end{aligned}
$$

If we compute the variation of the discrete energy, we obtain $\Delta E=E^{n+1 / 2}-E^{n-1 / 2}=\frac{1}{2} \sum_{i, j} \sum_{k=0}^{n-1} F(k) \Delta t \Delta x \Delta y$,

Where

$$
F(k)= \begin{cases}g\left(t^{1}\right) U_{i, j}^{n-1}\left(\Delta U_{i, j}^{n-1}-\Delta U_{i, j}^{n}\right) & , k=n-1, \\ {\left[g\left(t^{n-k}\right) U_{i, j}^{n-1}-g\left(t^{n-k-1}\right) U_{i, j}^{n}\right] \Delta U_{i, j}^{k}} & , k=0, \ldots, n-2 .\end{cases}
$$

The quantity $\Delta \mathrm{E}$ is numerically strictly negative as expected.

\section{Decay behavior of the discrete energy}

In order to show the non-increasing behavior of $\mathrm{g}$ and the decay relation $\mathrm{E}\left(\mathrm{t}^{\mathrm{n}}\right) \leq \operatorname{Eps}\left(\mathrm{t}^{\mathrm{n}}\right)$, we consider the examples stated in Section 3 and choose the following parameters:

$\alpha=0.3, \mathrm{Nx}=\mathrm{Ny}=50, \mathrm{~T}=10, \mathrm{k}=0.01, \mathrm{~K}=4, \mathrm{c}_{0}=0.01, \mathrm{a}=0.1, \mathrm{~b}=1.1$.

The space step is $\Delta \mathrm{x}=\Delta \mathrm{y}=0.1$ and the time step $\Delta \mathrm{t}=0.033$ (Figure 1).

\section{Conclusions}

In this work, we have the following conclusions

- The general decay result, known for viscoelastic problems with regular kernels, has been extended successfully to problems with singular kernels.

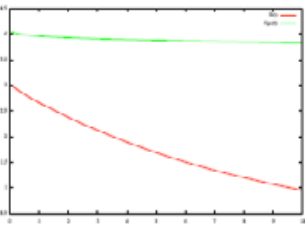

(a) Example 1

(c) Example 3

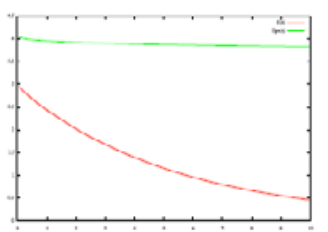

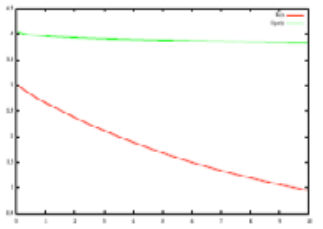

(b) Example 2

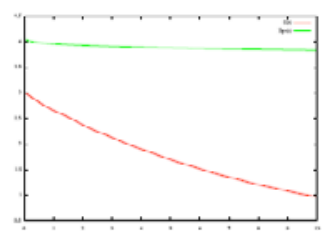

(d) Example 4
Figure 1: Energy decay examples 
Citation: Messaoudi SA, Khenous HB (2015) A General Stability Result for Viscoelastic Equations with Singular Kernels. J Appl Computat Math 4: 221. doi:10.4172/2168-9679.1000221

Page 5 of 5

- The decay result is established with weaker conditions on the function $\xi(\mathrm{t})$.

- The exponential decay results of $[33,34]$ are only special cases.

- The numerical tests presented for the four types of relaxation functions are in accordance with our theoretical result.

\section{Acknowledgment}

This work is sponsored by KFUPM-Project IN141015.

\section{References}

1. Desch W, Grimmer R (1989) Singular relaxation moduli and smoothing in three-dimensional viscoelasticity. Trans Amer Math Soc 314: 381-404.

2. Fabrizio M, Polidoro S (2002) Asymptotic decay for some differential systems with fading memory. Appl Anal 81: 1245-1264.

3. Alabau-Boussouira F, Cannarsa P, Sforza D (2008) Decay estimates for second- order evolution equations with memory. J Funct Anal 254: 1342-1372.

4. Barreto RK, Rivera JM, (1996) Uniform rates of decay in nonlinear viscoelasticity for polynomial decaying kernels. Appl Anal 60: 263-283.

5. Berrimi S, Messaoudi SA (2006) Existence and decay of solutions of a viscoelastic equation with a nonlinear source. Nonlinear Anal TMA 64: 23142331.

6. Cavalcanti MM, Cavalcanti VND, Filho JSP, Soriano JA (2001) Existence and uniform decay rates for viscoelastic problems with nonlinear boundary damping. Differential Integral Equations 14: 85-116

7. Cavalcanti MM, Cavalcanti VND, Soriano JA (2002) Exponential decay for the solution of semi linear viscoelastic wave equations with localized damping. Elect J Diff Eqns 44: 1-14.

8. Cavalcanti MM, Oquendo HP (2003) Frictional versus viscoelastic damping in a semi linear wave equation. SIAM J Control Optim 42: 1310-1324.

9. Cavalcanti MM, Cavalcanti VND, Martinez P (2008) General decay rate estimates for viscoelastic dissipative systems. Nonlinear Anal 68: 177-193.

10. Hrusa WJ (1985) Global existence and asymptotic stability for a semi linear Volterra equation with large initial data. SIAM J Math Anal 16: 110-134.

11. Hrusa WJ, Renardy M (1985) On wave propagation in linear viscoelasticity. Quarterly Appl Math 43: 237-254.

12. Hrusa WJ, Renardy M (1986) On a Class of Quasilinear Partial Integrodifferential Equations with Singular Kernels. J Diff Equations 64: 195-220.

13. Han Xi, Wang M (2009) General decay of energy for a viscoelastic equation with nonlinear damping. Math Meth Appl Sci 32: 346-358.

14. Munoz Rivera JE (1994) Asymptotic behavior in linear viscoelasticity. Quart Appl Math 52: 628-648.

15. Munoz Rivera JE, Peres Salvatierra A (2001) Asymptotic behavior of the energy in partially viscoelastic materials. Quart Appl Math 59: 557-578.

16. Munoz Rivera JE, Naso MG, Vegni FM (2003) Asymptotic behavior of the energy for a class of weakly dissipative second-order systems with memory. $J$ Math Anal Appl 286: 692-704

17. Munoz Rivera JE, Naso MG (2006) On the decay of the energy for systems with memory and indefinite dissipation. Asympt Anal 49: 189-204.

18. Mustafa MI, Messaoudi SA (2012) General stability result for viscoelastic wave equations. J Math Physics 53.

19. Park J, Park S (2009) General decay for quasilinear viscoelastic equations with nonlinear weak damping. J Math Physics 50.

20. Milota J, Necas J, Sverak V (1990) On weak solutions to a viscoelasticity model, Comment. Math Univ Carolinae 31: 557-565.
21. Lasiecka I, Messaoudi SA, Mustafa MI (2013) Note on intrinsic decay rates for abstract wave equations with memory. J Math Physics 54: 1-18.

22. Messaoudi SA (2008) General decay of solutions of a viscoelastic equation. $J$ Math Anal Appl 341: 1457-1467.

23. Messaoudi SA (2008) General decay of the solution energy in a viscoelastic equation with a nonlinear source. Nonlinear Anal TMA 69: 2589-2598.

24. Rouse PE (1953) A theory of the linear viscoelastic properties of dilute solutions of coiling polymers. J Chem Phys 21: 1271-1280.

25. Ye X, Tang G (2015) Effect of coupling asynchronous acoustoelectric effects on the corrosion behavior, microhardness and biocompatibility of biomedical titanium alloy strips. Journal of Materials Science: Materials in Medicine 26 : 1-15.

26. Renardy M (1982) Some remarks on the propagation and non-propagation of discontinuities in linearly viscoelastic liquids. Rheol Acta 21: 251-254.

27. Wu ST (2012) Exponential decay for a nonlinear viscoelastic equation with singular kernels. Acta Mathematica Scientia 32: 2237-2246.

28. Liu WJ (2009) General decay rate estimate for a viscoelastic equation with weakly nonlinear time-dependent dissipation and source terms. J Math Physics 50 .

29. Koeller RC (1984) Applications of fractional calculus to the theory of viscoelasticity. ASME J Appl Mech 51: 299-307.

30. Gentili G (1995) Regularity and stability for a viscoelastic material with a singular memory kernel. J Elasticity $37: 139-156$

31. Tatar NE (2009) Exponential decay for a viscoelastic problem with a singular kernel. Z Angew Math Phys 60: 640-650.

32. Laun HM (1978) Description of the nonlinear shear behavior of a low density polyethylene melt by means of an experimentally determined strain dependent memory function. Rheol Acta 17: 1-15.

33. Ye X, Tse ZTH, Tang G (2015) Mechanical properties and tensile fracture of Ti-Al-V alloy strip under electropulsing-induced phase change. J Mater Res 30: 206-223.

34. Xiaosen H, Mingxing W (2009) General decay of energy for a viscoelastic equation with nonlinear damping, Math. Methods Appl Sci 32: 346-358.

35. Carillo S, Valente V, Caffarelli GV (2010) A result of existence and uniqueness for an integro-differential system in magneto-viscoelasticity. Applicable Analysis 90: 1791-1802.

36. Carillo S, Valente V, Caffarelli GV (2012) An existence theorem for the magnetic viscoelastic problem. Discrete and Continuous Dynamical Systems Series S 5: 435-447.

37. Carillo S, Valente V, and Caffarelli GV (2013) A linear viscoelasticity problem with a singular memory kernel: an existence and uniqueness result. Differential and Integral Equations 26: 1115-1125.

38. Berti $\vee(2006)$ Existence and uniqueness for an integral-differential equation with singular kernel. Boll Un Mat Italiana Sez B 9-B: 299-309. 